\title{
Downregulation of Immune Response- and External Stimulus-Related Genes in CRC Organoids, and Their Re-Expression by Co-Culturing with CAFs
}

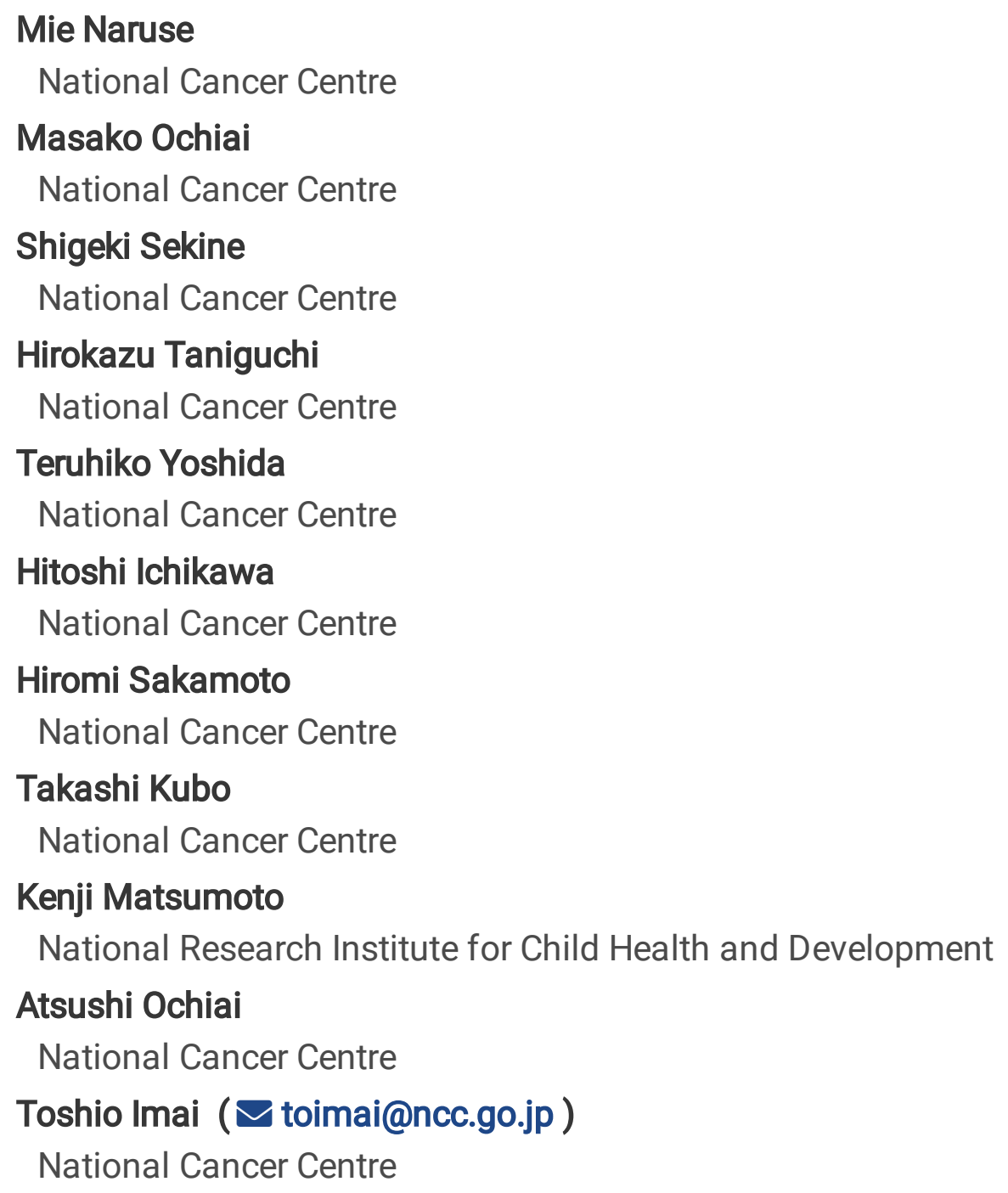

\section{Research Article}

Keywords: CAFs, REG, CRC, cancer

Posted Date: November 24th, 2020

DOI: https://doi.org/10.21203/rs.3.rs-107047/v1 
License: (c) (i) This work is licensed under a Creative Commons Attribution 4.0 International License. Read Full License

Version of Record: A version of this preprint was published at Scientific Reports on January 22nd, 2021. See the published version at https://doi.org/10.1038/s41598-021-81475-2. 


\section{Abstract}

Organoids derived from epithelial tumors have recently been utilized as a preclinical model in basic and translational studies. This model is considered to reproduce the features of cell-cell contacted and differentiated original tumor cells, but not the tumor microenvironment. In this study, we established organoids and paired cancer-associated fibroblasts (CAFs) from surgical specimens of colorectal carcinomas (CRCs), and evaluated gene expression profiles in organoids with and without co-culture with CAFs to assess interactions between tumor cells and CAFs in tumor tissues. We found that the expression levels of several genes, which are highly expressed in original CRC tissues, were downregulated in organoids but re-expressed by co-culturing with CAFs. They comprised immune response- and external stimulus-related genes, e.g., REG family and dual oxidases (DUOXs), which are known to have malignant functions, e.g., cell-proliferation and/or reducing apoptosis of epithelia and drug resistance for anti-cancer drugs in tumors. In addition, the degree of re-production of REG1 and DUOX2 in the co-culture system varied depending on CAFs from each CRC case. In conclusion, the coculture system of CRC organoids with paired CAFs was able to partially reproduce the tumor microenvironment.

\section{Introduction}

Two-dimensional (2D) cancer cell lines that can be easily and reproducibly maintained in vitro have been mainly used for cancer studies targeting the clarification of molecular mechanisms of carcinogenesis or evaluation of anticancer drugs. As most cancer cell lines were cultured under over-nourished conditions, differing from the in vivo environment, and repeated passages, the characteristics of original tumor tissues, such as expression of stem cell markers and differentiation marker genes, and proliferation, invasion, and drug metabolic abilities are altered in cancer cell lines ${ }^{1-3}$. The low probability of success of clinical trials is partly due to the use of cancer cell lines in preclinical evaluations of drug candidates ${ }^{3-6}$. To promote efficient drug discovery, organoid culture systems have been utilized recently 7,8 .

An organoid is a miniaturized and simplified organ produced in vitro using 3D culture systems that resembles a realistic micro-organ. They are derived from one or a few cells from a tissue, and demonstrate natural cell-cell communication and undergo self-organization in 3D culture ${ }^{9,10}$. As an ex vivo model, cancer tissue-derived organoids are considered suitable to analyze direct reactivities of carcinoma cells to growth factors/cytokines, miRNAs, or synthetic compounds; however, stroma cells, including fibroblasts, immune cells, and vascular cells, which interact with carcinoma cells in original cancer tissues, are absent in organoid systems. The interactions between cancer cells and cancer associated fibroblasts (CAFs) vary and are considered to be important for carcinogenesis ${ }^{11-17}$. Moreover, the epithelial mesenchymal transition (EMT), which enables tumor cells to acquire resistance to anticancer drugs, was reported to be facilitated by the presence of CAFs ${ }^{18-20}$. To date, there have been limited investigations using co-culture systems of 3D-cultured cancer cells with CAFs on several types of cancers, e.g., pancreatic ductal adenocarcinomas ${ }^{21,22}$, prostate adenocarcinomas ${ }^{23}$, and esophageal 
carcinomas ${ }^{24}$, and interactions between cancer cells and CAFs were demonstrated to affect cancer aggressiveness and resistance to anti-cancer drugs.

The purpose of the present study was to clarify if the co-culture system of CRC organoids with CAFs reproduces the microenvironment between tumor cells and CAFs observed in the original cancer tissues. First, comprehensive gene expression analyses between original tumor tissues and organoids were performed to characterize the features of the organoid culture system. Second, to identify gene expression changes in organoids induced by co-culturing with CAFs, paired CAFs from each case were established, and gene expression profiles between organoids with or without associated CAFs were compared. The present comparative screening of gene expression profiles of clinical tissue-derived CRC organoids with or without corresponding patient tissue-derived CAFs is highly important because the major molecules involved in interactions between tumor cells and CAFs have not been comprehensively evaluated in natural cell-cell communication-constructed and self-organizing in vitro systems.

\section{Results}

\section{Baseline characteristics of CRC}

Basic patient and pathological data in the $45 \mathrm{CRC}$ cases used for establishment of organoids and fibroblasts are summarized in Supplementary Table S1. Of the 45 cancer patients, $60 \%$ were males and $40 \%$ were females. This male per female ratio is comparable with Japanese Cancer Statistics (Cancer Registry and Statistics. Cancer Information Service, National Cancer Center, Japan (Monitoring of Cancer Incidence in Japan (MClJ)). Average ages were 62 years old, ranging from 36 years old to 88 years old. The proportions of stage I, stage II, stage III, and stage IV cancers were $7 \%, 47 \%, 31 \%$, and $15 \%$, respectively, and $85 \%$ were found in the left colon ( $D, S, R s, R a$, and $R b)$ and $15 \%$ in the right colon $(T, A$, and $\mathrm{C}$ ). The detailed baseline characteristics of donor patients are summarized in Supplementary Table S1 and Supplementary Fig. S1.

\section{Establishment of organoids and fibroblasts}

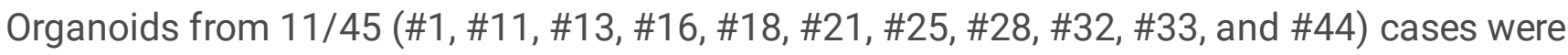
established. All CRC organoids had globular and/or irregularly elongated crypts with monolayered or partially multilayered structures. These features of organoids resembled those of CRC tumor tissues in cases \#21, \#28, and \#32 (Fig. 1a-f). CRC-derived cancer associated fibroblasts (CAFs) (Fig. 1g-i) and normal mucosa-derived fibroblasts (NFs) were isolated from cancer tissues and adjacent normal tissues, respectively. The success rate of the presently established organoids was $24.4 \%(11 / 45)$, that of CAFs was $77.1 \%(27 / 35)$, and that of NFs was $88.6 \%$ (31/35). CAFs were established from cases obtained during the period from September 2016 to February 2018. The success rate of CRC-derived organoids did not correlate with the basic patient or pathological information, e.g., the sex, age of patient, or pathological stage of cancer (Supplementary Table S2). The characteristic difference between CAFs and NFs was the high expression levels of actin alpha 2, smooth muscle (ACTA2), a marker for CAFs, in cases 
\#28 and \#32 (Fig. 1j). In case \#21, NFs were not available; however, the expression level of ACTA2 in CAFs was comparable with that in CAFs from \#28 and \#32.

\section{Mutation analysis by NCC Oncopanel}

Mutations in original CRC tissues and established organoids were analyzed by targeted sequencing using the NCC Oncopanel test. The prevalence rates of APC, TP53, and KRAS mutations in original tissues were $91.1 \%, 80.0 \%$, and $35.6 \%$, respectively (Fig. 2). The frequently mutated genes including APC, TP53, and KRAS were highly consistent between our cohort and TCGA (The Cancer Genome Atlas) database 25 . Most of the mutations found in the CRC tissues were maintained in their organoids with higher mutation allele frequencies. However, there are some exceptions, suggesting a reflection of intratumor heterogeneity. Loss of the minor mutations in organoids was considered to partly be attributed to clonal evolution of cancer cells during the organoid culture (Table 1).

Then, we performed Sanger sequencing to confirm that the established fibroblasts did not harbor the mutations found in cancer tissues. The TP53 mutations of the cases \#21, \#28, and \#32 were detected in their organoids but not in their fibroblasts (Supplementary Fig. S2). Other mutations such as those of $D D R 2$ in \#21, ESR 1 in \#28, and APC in \#32, were also not detected in the corresponding fibroblasts (data not shown).

\section{Gene expression analysis by DNA microarray}

Gene expression analyses by DNA microarray were carried out using original CRC specimens and organoids. Data sets for original tumor tissues and organoids from 5 cases were successfully adjusted and validated for principal components analysis (PCA). The PC1-axis demonstrated a different spatial distribution between the original tumors and organoids. The two groups had significant differences in gene expression distribution, indicating two transcriptionally distant populations (Fig. 3a). There were two typical gene expression profiles of PC1 genes: One was the gene groups highly expressed in organoids and the other was gene groups whose expression in organoids was lower than that in the original tumors. In total, 586 probes exhibited a similar gene expression profile with the latter group of PC1 genes (Pearson's correlation between 0.95 and 1). Based on Gene Ontology (G0) term analysis, the genes with lower expression in organoids than in the original tumors were enriched for GO terms "extracellular matrix organization", "blood vessel development", and "lymphocyte activation" (Fig. 3c). This reflected the lack of fibrous, vascular, and immune cell populations in organoids, whereas the original tumors consist of both epithelial cells and stromal cells. On the other hand, expression profiles of intestinal epithelialstemness-related genes demonstrated that the organoids maintained the characteristics of the original CRC tissues (Fig. 3d). It is also possible that there was a subset of genes induced via cell-cell communication with other cell types, including CAFs.

\section{Cell proliferation abilities of CRC organoids were stimulated by co-culturing with paired CAFs}


The cell proliferation ability significantly varied among CRC organoid lines (Fig. 4a). CAFs were also established from the same CRC specimens in some cases. To address the effects of cell-cell interaction between CRC organoids and corresponding CAFs, we developed a novel co-culture method using a chamber system. By co-culturing organoids with CAFs, cell viability of organoids increased by 1.2 to 1.5 fold compared with corresponding single-culture organoids in three out of four cases (Fig. 4a). This suggested that the tumor microenvironment constructed by cell-cell communication between tumor cells and CAFs plays a role in the cell proliferative/anti-apoptotic abilities of tumor cells.

\section{Identification of upregulated genes by co-culturing with CAFs}

To identify genes whose expression levels were different between organoids co-cultured with and without CAFs, we performed DNA microarray analysis. Volcano plot analysis demonstrated that the expression levels of 73 genes with 87 probes in organoids were reduced to less than half, whereas those of 177 genes with 238 probes were increased by more than double by co-culturing with CAFs $(P<0.05$; Fig. 4b, Supplementary Tables S3 and S4). These upregulated genes included REG1A, REG1B, REG3A, REG3G, $D M B T 1$, DUOXA2, DUOX2, SOCS3, and LOC340340, with more than 10-fold upregulation. Hierarchical clustering analysis confirmed that these CAF-induced genes were expressed at higher levels also in the original CRC tissues but not in single-culture organoids (Fig. 4c). To validate the microarray data obtained from the co-culture of CRC organoids and CAFs, two candidates for CAF-induced gene groups, REG $1 A$, REG3A, DUOX2, and DUOX2A, were quantified by quantitative RT-PCR (Fig. 4d-g). The levels of REG1A increased by more than 121 (\#21), 480 (\#28), and 21-fold (\#32) when co-cultured with CAFs. The levels of REG3A increased by more than 26 (\#21), 55 (\#28), and 11-fold (\#32). The levels of DUOX2 increased by more than 29 (\#21), 3 (\#28), and 33-fold (\#32). The levels of DUOXA2 increased by more than 10 (\#21), 3 (\#28), and 15-fold (\#32). This analysis confirmed that the microarray data is reliable, and the degree of induction of each REG family and dual oxidase gene by CAFs varied among cases.

Other than $R E G$ family and dual oxidase genes, microarray analysis suggested that expression of CEACAM6 and CEACAM7, members of the carcinoembryonic antigen-related cell adhesion molecule family, and MUC1, which inhibits the anti-tumor immune response, was upregulated by more than 2-fold (Supplementary Table S3).Although many cancer-related genes were induced by the co-culture with CAFs, the degree of their induction by CAFs varied among cases.

GO term enrichment analysis for upregulated genes in organoids by co-culturing with CAFs revealed that innate immune response, including "cell wall disruption in other organisms", "response to bacterium", "complement and coagulation cascades", and "interferon-gamma-mediated signaling pathway", were significantly enriched (Supplementary Fig. S3a). These induced pathways by CAFs were abundantly expressed in CRC tissues but downregulated in single-culture CRC organoids (Supplementary Fig. S3b).

\section{CAFs derived from different cases exhibit case-specific ability for REG1A induction in organoids}

Organoids derived from different cases exhibited varying degrees of induction of REG family and dual oxidase genes by co-culturing with paired CAFs, e.g., organoids from cases \#21 and 32 had high 
reactions for dual oxidase genes, and those from case \#28 had high reactions for REG family genes (Fig. $4 \mathrm{~d}-\mathrm{g}$ ). Next, combinations of organoids and CAFs derived from different cases were examined because

CAFs consist of numerous cell types and their characteristics vary among lines ${ }^{26,27}$. Organoids of cases \#28 and \#32 co-cultured with CAFs from three different cases and corresponding normal mucosa-derived fibroblasts (NFs) exhibited almost identical induction patterns by co-culture with CAFs from each case and corresponding NFs (Fig. 5a and b), suggesting the case-specific ability of CAFs for the induction of REG1A in organoids. Thus, re-expressed genes in CRC organoids by co-culturing with CAFs may depend on the characteristics of each organoid and CAF line.

\section{Discussion}

We established 11 organoids from 45 CRC cases, and simultaneously prepared paired CAFs and normal fibroblasts from several of them. Interactions between cancer cells and CAFs using colon or lung cancer cell line-originated organoids and patient-derived CAFs were previously reported ${ }^{28,29}$. Other reports suggested that the molecular characteristics of CAFs vary among cases ${ }^{30,31}$. The newly established CRC model of organoids co-cultured with paired CAFs from the same case in the present study may provide novel insights into cell-cell communication between these cell types.

Target sequencing analyses of original tumor tissues revealed that mutation patterns varied among patients, and the most frequently mutated genes were $A P C, T P 53$, and KRAS, as previously reported ${ }^{25}$. In addition, most mutations found in the original tumor tissues were conserved in organoids ${ }^{32}$. The mutation rates in CRC organoids were higher than those in CRC tissues, with several exceptions (Table 1). This may be because CRC organoids consist of only epithelial tumor cells, whereas CRC tissues include epithelial tumor cells and stromal cells, which do not have mutations. Indeed, CAFs did not carry the mutations found in corresponding CRC organoids (Supplementary Fig. S2). The fold changes in the mutation ratios (early passage/late passage - up to passage 20) were almost identical (0.84-1.13) (Table 1 ), as previously reported in ovarian cancer organoids ${ }^{33}$. This suggests that organoids from CRC passaged up to 20 times can be applied as an ex vivo model in basic and translational studies for the evaluation of anti-cancer drugs. Furthermore, cancer stem cells comprising CRC organoids and their proliferative ability were maintained in our culture conditions.

Next, to examine whether CRC organoids can reproduce the gene expression of the original tumor tissues, gene expression profiles were compared between the original tumor tissues and established CRC organoids using DNA microarray (Fig. 3). As a result, CRC organoids lacked gene expression from mesenchymal cell populations and blood cells, whereas each CRC organoid had a variable expression profile for intestinal stem cell marker genes, e.g. LGR5 and ORFM4, as observed in CRC tissues. This suggests that gene expression in CRC organoids is mostly conserved, but they lack cell-cell communication with the tumor microenvironment, including fibroblasts and immune cells.

In this study, a co-culture system using the CRC organoids and paired CAFs and/or normal fibroblasts from each case was prepared, and gene expression profiles for both CRC organoids with or without co- 
culture with CAFs were compared. As a result, we identified 177 genes, including REG family and DUOX family genes, which were markedly upregulated by more than 2 -fold by co-culturing with CAFs in all three cases analyzed. Some of these upregulated genes were reported to have oncogenic functions. For example, REG family genes are known to be upregulated in several carcinomas compared with normal tissue ${ }^{34-37}$. REG family genes have cell-proliferative and anti-apoptotic functions ${ }^{38}$. REG3A is also known to act as an extracellular matrix (ECM)-targeted scavenger of reactive oxygen species (ROS) in a dose-dependent manner and to prevent ROS-induced mitochondrial damage due to acetaminophen overdose $^{39,40}$. ROS is a key regulator for EMT, and is produced by NOX gene family and DUOX family genes ${ }^{41}$. Therefore, REG3A and DUOX2 gene expression is considered to be important for EMT. DUOX family genes also have cell-proliferative functions ${ }^{41,42}$. Moreover, DUOX2 was reported to have anticancer drug resistant activities through EMT ${ }^{43}$. GO term enrichment analysis for the above mentioned 177 upregulated genes revealed that co-culturing of CRC organoids with CAFs induced innate immune responses, suggesting that some oncogenic signal cascades induced by cell-cell interaction between organoids and CAFs were mediated by the signal pathways related to immune responses.

Other than immune response- and external stimulus-related genes, CEACAM6, a member of the carcinoembryonic antigen (CEA) family ${ }^{44}$, was also upregulated by more than 2 -fold in CRC organoids by co-culturing with CAFs. CEA is normally produced in gastrointestinal tissue during fetal development, but its production stops before birth. Consequently, CEA is usually present at low levels in the blood of healthy adults (approximately $2-4 \mathrm{ng} / \mathrm{mL}$ ) ${ }^{45}$. However, the serum levels increase in some types of cancer, suggesting its utility as a diagnostic marker and/or a drug efficacy marker in clinical tests. CEACAM6 was highly upregulated in colon cancer tissues and may therefore be a suitable candidate for a diagnostic marker of colorectal cancer ${ }^{46}$. CEACAM6 loss increases mitochondrial basal and maximal respiratory capacity. It also affects several hallmarks of pancreatic ductal adenocarcinoma (PDA), including fibrotic reactions, immune regulation, and energy metabolism, and was recently investigated as a novel therapeutic target in PDA ${ }^{47}$. Thus, there is a subset of genes expressed in CRC tumor cells in the original tissues, but not in the CRC organoids without co-culture with CAFs. These silenced genes in CRC organoids were induced by the co-culture with CAFs (Fig. 4c). The degrees of upregulation of the genes by co-culturing with CAFs varied among the CRC cases. We investigated whether these effects on the gene expression changes depend on the ability of CAFs or their compatibility with CRC organoids. Organoids from two CRC cases exhibited almost identical induction patterns by co-culture with CAFs from three different cases and paired normal mucosa-derived fibroblasts (Fig. 5). Therefore, the degrees of upregulation of several genes, e.g., REG1A, depend on the ability of CAFs from CRC. In addition, it should be noted that normal fibroblasts also induced gene expression, suggesting that some factors, possibly growth factors/cytokines secreted by CRC organoids or miRNA delivered by extracellular vesicles (EVs) secreted from CRC organoids, can induce gene expression to a similar degree as CAFs. To address which factors are essential for the induction of genes in CRC organoids, detailed analyses of the co-culture system of CRC organoids are needed. The upregulated genes mentioned above were related to malignancy of tumor cells, and the malignancy of cancer may partly depend on the characteristics of 
CAFs and/or original normal fibroblasts. Indeed, cell viability of organoids increased by 1.2 to 1.5 -fold compared with corresponding single-culture organoids in three of four cases by co-culturing with CAFs (Fig. 4a); however, those in one case (\#28) were not altered. We compared the efficiency of induction of the REG1A gene in organoids by co-culture with CAFs from three cases and it was the lowest by \#28 CAFs. In conclusion, 1) gene mutation patterns, fold changes in the mutation allele frequencies, and expression profiles for intestinal stem cell marker genes were nearly similarly maintained in CRC organoids as in the original tumor tissues, suggesting that CRC organoids can be applied as an ex vivo model in basic and translational studies. 2) Expression levels of several genes, which are highly expressed in original CRC tissues, were downregulated in organoids but re-expressed by co-culturing with CAFs. They comprised immune response- and external stimulus-related genes, e.g., REG family and dual oxidases (DUOXs). In addition, gene induction by co-culturing with CAFs varied depending on the CAFs from each $\mathrm{CRC}$ case, suggesting that the present co-culture system of CRC organoids with paired CAFs partially reproduces the tumor-microenvironment.

\section{Materials And Methods}

\section{Tissue sampling}

A total of 45 surgical specimens of colorectal carcinomas (CRCs) and adjacent normal mucosa were obtained between February 2016 and February 2018 at National Cancer Center Hospital. All experimental methods were carried out in accordance with relevant guidelines and regulations. The use of patients' surgical specimens in this study was approved by the ethics committee of the National Cancer Center, Tokyo, Japan (2015-108), and written informed consent was obtained from all patients. After sampling for pathological evaluation, they were stored on ice, and each sample was dissected into approximately 2-5-mm cubes and used for culture of organoids and fibroblasts. Remaining fragments were simultaneously frozen in liquid nitrogen and stored at $-80^{\circ} \mathrm{C}$ for isolation of DNA and RNA, or fixed with $10 \%$ buffered formalin for preparation of tissue sections for morphological identification of the organoidand fibroblast-originated tumor tissues. The fragments for RNA isolation were stored in RNA/ater solution (ThermoFisher Scientific, Tokyo, Japan) at $4^{\circ} \mathrm{C}$ overnight before storage.

\section{Organoid culture}

The protocol employed for organoid culture was a modified version of those previously reported 48,49 . The fragments of CRC tissues were washed with cold HBSS(-), minced with scissors, and washed again. These fragments were incubated in Accumax (Innovative Cell Technologies, San Diego, CA, USA) at room temperature or TrypLE Express (ThermoFisher Scientific, Tokyo, Japan) at $37^{\circ} \mathrm{C}$ for $30 \mathrm{~min}$ with shaking. The digested fragments were rinsed with cold HBSS(-), dissociated using a 100- $\mu \mathrm{m}$ cell strainer, and pelleted. Advanced DMEM/F12 (ThermoFisher Scientific) supplemented with 1 x penicillin-streptomycin, $500 \mathrm{ng} / \mathrm{ml}$ of Amphotericin B (FUJIFILM Wako, Osaka, Japan), 1 x Zell Shield (Minelva Biolabs GmbH, Berlin, Germany), 10 mM HEPES (ThermoFisher Scientific), 1 x L-glutamine (FUJIFILM Wako), [Leu ${ }^{15}$ ]Gastrinl (Sigma-Aldrich, Tokyo, Japan), 1 mM N-acetyl-L-cysteine (FUJIFILM Wako), 1 x B27 supplement 
(ThermoFisher Scientific), 1\% BSA (FUJIFILM Wako), and $10 \mu \mathrm{M}$ Y27632 (FUJIFILM Wako) was used as the basal culture medium for CRC organoids. The pellet was suspended in the basal culture medium containing the following factors: $50 \mathrm{ng} / \mathrm{ml}$ of Recombinant Human EGF (Peprotech, Rocky Hill, NJ, USA), $100 \mathrm{ng} / \mathrm{ml}$ of Noggin (Peprotech), and 500 nM A83-01 (FUJIFILM Wako).

In a 12-well plate, $65 \mu$ l of Matrigel (Corning, Bedford, MA, USA) / well was polymerized for 15 min at $37^{\circ} \mathrm{C}$ and a $650-\mu \mathrm{l}$ cell suspension / well $\times 3$ was seeded and incubated in a $37^{\circ} \mathrm{C}$ humidified $\mathrm{CO}_{2}$ incubator. After 24 hours, the supernatants were removed and $85 \mu \mathrm{l} /$ well of Matrigel was overlaid. After Matrigel polymerization, the basal culture media containing different factor combinations [A:250 ng/ml of Rspondin 1 (Peprotech) +20 ng/ml of Wnt-3a (Peprotech) $+25 \mu$ M SB202190, B: $25 \mu$ M SB202190, and C: none] was used to select the most efficient growth media during several passages. The organoids were dispersed by Accumax and passaged approximately once a week. Zell Shield was not used after several passages. When organoids with more than 10 passages (P10) survived after a freeze and thaw cycle, they were defined as "successfully established".

\section{Fibroblast culture}

Two to three tissue fragments were washed three times with HBSS(-), placed in a 60-mm dish, and minced with scissors. Then, culture medium, RPMI-1640, containing L-Glutamine (FUJIFILM Wako) and $10 \%$ FBS, penicillin-streptomycin was added into the dish and cells were incubated at $37^{\circ} \mathrm{C}$ in a humidified $5 \% \mathrm{CO}_{2}$ incubator. After they reached $70 \%$ confluency, cells were passaged using TrypLE Express dissociation reagent (ThermoFisher Scientific).

\section{Co-culture of organoids with fibroblasts using a chamber system}

Organoids were cultured in the cell culture inserts with a porous membrane and fibroblasts were cultured in the carrier plates (Corning). The pore size of the insert was $1.0 \mu \mathrm{m}$ to allow the free exchange of media but not cells to migrate through. One day before starting the co-culture, fibroblasts were dissociated into single cells using TrypLE Express and $1 \times 10^{4}$ cells were cultured in a 24-well plate with RPMI-1640 containing penicillin-streptomycin, Amphotericin B, and 10\% FBS. For organoid culture, cell culture inserts were set on the 24-well companion plate and $20 \mu \mathrm{l}$ of Matrigel was polymerized on the insert. Organoids were dissociated by Accumax and resuspended in optimized media for organoids described above. The cell suspension ( $1 \times 10^{4}$ cells / $200 \mu \mathrm{l}$ ) was seeded onto the Matrigel and $620 \mu \mathrm{l}$ of media for organoids was added to the basal compartment. Fibroblasts and organoids were incubated at $37^{\circ} \mathrm{C}$ in a $\mathrm{CO}_{2}$ incubator.

The next day, apical and basal media were removed from the plate containing organoids attached on the Matrigel, and organoids were covered with $20 \mu \mathrm{l}$ of Matrigel and polymerized at $37^{\circ} \mathrm{C}$ for $15 \mathrm{~min}$. The inserts containing organoids were transferred to the fibroblast-containing compartment plate after changing the medium from that for fibroblasts to $820 \mu \mathrm{l}$ of optimized media for organoids. The co-culture 
plate was incubated at $37^{\circ} \mathrm{C}$ in a $\mathrm{CO}_{2}$ incubator for 96 hours, and organoids and fibroblasts were collected separately for gene expression analysis.

\section{Targeted sequencing analysis}

Genomic DNA from 45 samples of CRC and organoids of cases \#11, \#21, \#25, \#28, \#32, \#33, and \#44 were prepared using NucleoSpin Tissue kit (Takara Bio, Kusatsu, Japan) according to the manufacturer's protocol. Targeted sequencing analyses of those DNAs were performed using the NCC Oncopanel v4 test, which can analyze mutations of 114 genes and amplifications and fusions of 12 genes ${ }^{50}$. Procedures for targeted sequencing and data analysis were previously described ${ }^{50}$.

TP53 mutations identified by the NCC Oncopanel test were confirmed by Sanger sequencing. The PCR products including mutations were amplified using specific PCR primers. Primers used for A578G and G589A mutations of \#21 and \#32, respectively, were forward: 5'-GGAGGTCAAATAAGCAGCAGG-3' and reverse: 5'-GGCCTCTGATTCCTCACTGA-3'. Primers used for a 45_48TCAG deletion of \#28 were forward: 5'-CCCAACCCTTGTCCTTACCA-3' and reverse: 5'-CAGTCAGATCCTAGCGTCGA-3'. The amplified PCR products were directly sequenced by Sanger sequencing using the following primers. The primer for \#21 and \#32 was 5'-ACAACCACCCTTAACCCCTC-3'. The primer for \#28 was 5'- CCCAACCCTTGTCCTTACCA $-3^{\prime}$

\section{Gene expression analysis using DNA microarray}

Total RNA was extracted from original tumor tissues and established organoids of cases \#11, \#25, \#28, \#32, and \#44 using NucleoSpin RNA Plus (TaKaRa) according to the manufacturer's protocol. Total RNA was extracted from three replicates of co-cultured organoids and fibroblasts for \#21, \#28, and \#32 using the RNeasy Micro kit (QIAGEN, Tokyo, Japan). The quality of the RNA samples was evaluated using an Agilent 2100 Bioanalyzer (Agilent Technologies, Santa Clara, CA, USA) and highly qualified RNA samples

with a RIN score $>7.0$ were selected for further DNA microarray analysis. Triplicate samples were used as an RNA cocktail. Cy3-labeled cRNA was hybridized on SurePrint G3 Human GE Microarray GE 8 x 60K Ver.3.0 (Agilent Technologies) according to the manufacturer's instructions. The Subio Platform, Subio Basic plug-in, and Subio Advanced plug-in (ver 1.24) (Subio, Kagoshima, Japan) were used for data analysis.

\section{Quantitative real-time PCR}

One $\mu \mathrm{g}$ of total RNA for each sample was treated with DNase I (NIPPON GENE, Tokyo, Japan) prior to reverse transcription to CDNA using Multiscribe Reverse Transcriptase with random primers (ThermoFisher Scientific). qRT-PCR was performed with SsoAdvanced Universal SYBR Green Supermix (BIO-RAD, California, USA) using DNA Engine Opticon 2 (MJ Research, Quebec, Canada). Reactions were run in triplicate in three independent experiments. Data were normalized with the housekeeping gene b$A C T I N$ and were calculated by the $2-\triangle \Delta C T$ method ${ }^{51}$. Data were presented as means $\pm S D$ (1.0-fold as the control). The primer sequences were as follows: b -ACTIN forward 5'- AAACTGGAACGGTGAAGGTG-3' 
and reverse AGAGAAGTGGGGTGGCTTTT3', ACTA2 forward 5'-CTGTTCCAGCCATCCTTCAT-3' and reverse 5'-GCTGGAAGGTGGACAGAGAG-3', REG1A forward 5' CTGGAATCCTGTGCTTGAGG-3' and reverse 5'GGTCTCCTACAAGTCCTGGG-3', REG3A forward 5'-CCTCTGGAAACCTGGTGTCT3' and reverse 5'CCACTCCCAACCTTCTCCAT3', DUOX2 forward 5'- GAGCCCTTCTTCAACTCCCT-3' and reverse 5'GGAGGACAGGCTCAGAAGTT-3', and DUOXA2 forward 5'-GGTCTCCTACAAGTCCTGGG-3' and reverse 5'TTTACAGATCGCCCCAGGAG-3'.

Cell viabilityThe viability of organoids was assessed after co-culture with fibroblasts for 96 hours using the chamber system. After aspirating culture medium, $100 \mu \mathrm{l}$ of fresh culture medium was added. Then, Matrigel including organoids was scraped using a mini cell scraper, $100 \mu \mathrm{l}$ of CellTiter-Glo 3D reagent was added (Promega, Tokyo, Japan), and organoids were disrupted by pipetting. Suspensions were transferred to 96 -well assay plates and incubated at room temperature for $25 \mathrm{~min}$. After incubation, the luminescence was measured using Synergy H1 (BioTek, Tokyo, Japan).

\section{Gene Ontology (GO) enrichment analysis}

To clarify the biological meaning of the key modules, the gene information was loaded into Metascape (http://metascape.org) for Gene Ontology (GO) enrichment analysis ${ }^{52}$. Terms with a $P$-value $<0.01$, a minimum count of 3 , and an enrichment factor $>1.5$ were collected and grouped into clusters based on their membership similarities (Kappa scores $>0.3$ ).

\section{Statistical analysis}

The associations between clinical factors and the establishment of organoids were tested using Fisher's exact test in EZR ${ }^{53}$. For qRT-PCR and cell viability assay, results were presented as the mean \pm s.d. Differences between groups were analyzed by the Student's- $t$ - test or one-way ANOVA using EZR. $P$ values of $<0.05$ were considered significant.

\section{Declarations}

\section{Acknowledgments}

We thank the Omics Core and Animal Core Facilities of the National Cancer Center Research Institute for the targeted sequencing analysis and their technical support in histopathological evaluations. The Core Facilities were supported by the National Cancer Center Research and Development Fund (2020-J-002). We are grateful to Ryoichi Masui, Ruri Nakanishi, Naoaki Uchiya, and Yurika Shiotani for their excellent technical assistance.

\section{Author contributions}

M.N. carried out overall experiments, the initial development of the methods for co-culture of organoids with fibroblasts, and drafted the submitted version of this manuscript. M.O. performed several of the initial organoid culturing. S.S. and H.T. collected the surgical specimens and evaluated pathological and 
clinical information. T.Y., H.I. H.S. and T.K. performed mutation analysis by NCC Oncopanel, and K.M. performed gene expression analysis by DNA microarray. A.O. contributed in developing the overall strategies and concepts. T.I. was responsible for the overall study design, data analysis and manuscript finalization.

\section{Funding}

This study was supported by a Grant for Research on Development of New Drugs and Funding for Research to Expedite Effective Drug Discovery by Government, Academia and Private Partnership (GAPFREE) from the Japan Agency for Medical Research and Development (AMED).

\section{References}

1. Birgersdotter, A., Sandberg, R. \& Ernberg, I. Gene expression perturbation in vitro-a growing case for three-dimensional (3D) culture systems. Semin Cancer Bio/15, 405-412 (2005).

2. Weaver, V.M. et al. Reversion of the malignant phenotype of human breast cells in three-dimensional culture and in vivo by integrin blocking antibodies. J Cell Bio/137, 231-245 (1997).

3. DiMasi, J.A. \& Grabowski, H.G. Economics of new oncology drug development. J Clin Onco/25, 209216 (2007).

4. Breslin, S. \& O'Driscoll, L. Three-dimensional cell culture: the missing link in drug discovery. Drug Discov Today18, 240-249 (2013).

5. Hopkins, A.L. Network pharmacology: the next paradigm in drug discovery. Nat Chem Bio/4, 682-690 (2008).

6. Kola, I. The state of innovation in drug development. Clin Pharmacol Ther83, 227-230 (2008).

7. Lancaster, M.A. \& Knoblich, J.A. Organogenesis in a dish: modeling development and disease using organoid technologies. Science345, 1247125 (2014).

8. Fang, Y. \& Eglen, R.M. Three-Dimensional Cell Cultures in Drug Discovery and Development. SLAS Discov22, 456-472 (2017).

9. Clevers, H. Modeling Development and Disease with Organoids. Cel/165, 1586-1597 (2016).

10. Drost, J. \& Clevers, H. Organoids in cancer research. Nat Rev Cancer18, 407-418 (2018).

11. Xouri, G. \& Christian, S. Origin and function of tumor stroma fibroblasts. Semin Cell Dev Bio/21, 40-46 (2010).

12. LeBleu, V.S. \& Kalluri, R. A peek into cancer-associated fibroblasts: origins, functions and translational impact. Dis Model Mech11 (2018).

13. Kati Räsänen, A.V. Activation of fibroblasts in cancer stroma. Experimental Cell Research316, 27132722 (2010).

14. Kalluri, R. The biology and function of fibroblasts in cancer. Nat Rev Cancer16, 582-598 (2016). 
15. Shiga, K. et al. Cancer-Associated Fibroblasts: Their Characteristics and Their Roles in Tumor Growth. Cancers (Basel)7, 2443-2458 (2015).

16. Kalluri, R. \& Zeisberg, M. Fibroblasts in cancer. Nat Rev Cancer6, 392-401 (2006).

17. Tommelein, J. et al. Cancer-associated fibroblasts connect metastasis-promoting communication in colorectal cancer. Front Onco/5, 63 (2015).

18. Shan, T. et al. Prometastatic mechanisms of CAF-mediated EMT regulation in pancreatic cancer cells. Int J Onco/50, 121-128 (2017).

19. Yu, Y. et al. Cancer-associated fibroblasts induce epithelial-mesenchymal transition of breast cancer cells through paracrine TGF-beta signalling. Br J Cancer110, 724-732 (2014).

20. Zhuang, J. et al. TGFbeta1 secreted by cancer-associated fibroblasts induces epithelialmesenchymal transition of bladder cancer cells through IncRNA-ZEB2NAT. Sci Rep5, 11924 (2015).

21. Ohlund, D. et al. Distinct populations of inflammatory fibroblasts and myofibroblasts in pancreatic cancer. J Exp Med214, 579-596 (2017).

22. Biffi, G. et al. IL1-Induced JAK/STAT Signaling Is Antagonized by TGFbeta to Shape CAF Heterogeneity in Pancreatic Ductal Adenocarcinoma. Cancer Discov9, 282-301 (2019).

23. Adisetiyo, $\mathrm{H}$. et al. Dependence of castration-resistant prostate cancer (CRPC) stem cells on CRPCassociated fibroblasts. J Cell Physio/229, 1170-1176 (2014).

24. Ebbing, E.A. et al. Stromal-derived interleukin 6 drives epithelial-to-mesenchymal transition and therapy resistance in esophageal adenocarcinoma. Proc Natl Acad Sci U S A116, 2237-2242 (2019).

25. Cancer Genome Atlas, N. Comprehensive molecular characterization of human colon and rectal cancer. Nature487, 330-337 (2012).

26. Ohlund, D., Elyada, E. \& Tuveson, D. Fibroblast heterogeneity in the cancer wound. J Exp Med211, 1503-1523 (2014).

27. Augsten, M. Cancer-associated fibroblasts as another polarized cell type of the tumor microenvironment. Front Onco/4, 62 (2014).

28. Dolznig, $\mathrm{H}$. et al. Modeling colon adenocarcinomas in vitro a 3D co-culture system induces cancerrelevant pathways upon tumor cell and stromal fibroblast interaction. Am J Patho/179, 487-501 (2011).

29. Nakamura, H. et al. Organoid culture containing cancer cells and stromal cells reveals that podoplanin-positive cancer-associated fibroblasts enhance proliferation of lung cancer cells. Lung Cancer134, 100-107 (2019).

30. Herrera, M. et al. Functional heterogeneity of cancer-associated fibroblasts from human colon tumors shows specific prognostic gene expression signature. Clin Cancer Res19, 5914-5926 (2013).

31. Chen, X. \& Song, E. Turning foes to friends: targeting cancer-associated fibroblasts. Nat Rev Drug Discov18, 99-115 (2019).

32. Weeber, F. et al. Preserved genetic diversity in organoids cultured from biopsies of human colorectal cancer metastases. Proc Natl Acad Sci U S A112, 13308-13311 (2015). 
33. Kopper, O. et al. An organoid platform for ovarian cancer captures intra- and interpatient heterogeneity. Nat Med25, 838-849 (2019).

34. Zheng, H.C. et al. Expression profile of the REG gene family in colorectal carcinoma. J Histochem Cytochem59, 106-115 (2011).

35. Matsumura, N. et al. Identification of novel molecular markers for detection of gastric cancer cells in the peripheral blood circulation using genome-wide microarray analysis. Exp Ther Med2, 705-713 (2011).

36. Nigri, J. et al. PAP/REG3A favors perineural invasion in pancreatic adenocarcinoma and serves as a prognostic marker. Cell Mol Life Sci74, 4231-4243 (2017).

37. Sasaki, Y. et al. REG1A expression is an independent factor predictive of poor prognosis in patients with breast cancer. Ann Surg Onco/15, 3244-3251 (2008).

38. Chen, Z., Downing, S. \& Tzanakakis, E.S. Four Decades After the Discovery of Regenerating IsletDerived (Reg) Proteins: Current Understanding and Challenges. Front Cell Dev Bio/7, 235 (2019).

39. Moniaux, N. et al. Human hepatocarcinoma-intestine-pancreas/pancreatitis-associated protein cures fas-induced acute liver failure in mice by attenuating free-radical damage in injured livers. Hepatology53, 618-627 (2011).

40. Lieu, H.T. et al. HIP/PAP accelerates liver regeneration and protects against acetaminophen injury in mice. Hepatology42, 618-626 (2005).

41. Lin, S.C. et al. High Immunoreactivity of DUOX2 Is Associated With Poor Response to Preoperative Chemoradiation Therapy and Worse Prognosis in Rectal Cancers. J Cancer8, 2756-2764 (2017).

42. Wang, J. et al. PKCalpha promotes generation of reactive oxygen species via DUOX2 in hepatocellular carcinoma. Biochem Biophys Res Commun463, 839-845 (2015).

43. Kang, K.A. et al. DUOX2-mediated production of reactive oxygen species induces epithelial mesenchymal transition in 5-fluorouracil resistant human colon cancer cells. Redox Bio/17, 224-235 (2018).

44. Rizeq, B., Zakaria, Z. \& Ouhtit, A. Towards understanding the mechanisms of actions of carcinoembryonic antigen-related cell adhesion molecule 6 in cancer progression. Cancer Sci109, 3342 (2018).

45. Ballesta, A.M., Molina, R., Filella, X., Jo, J. \& Gimenez, N. Carcinoembryonic antigen in staging and follow-up of patients with solid tumors. Tumour Bio/16, 32-41 (1995).

46. Kim, K.S. et al. Overexpression and clinical significance of carcinoembryonic antigen-related cell adhesion molecule 6 in colorectal cancer. Clin Chim Acta415, 12-19 (2013).

47. Pandey, R. et al. Carcinoembryonic antigen cell adhesion molecule 6 (CEACAM6) in Pancreatic Ductal Adenocarcinoma (PDA): An integrative analysis of a novel therapeutic target. Sci Rep9, 18347 (2019).

48. Fujii, M., Matano, M., Nanki, K. \& Sato, T. Efficient genetic engineering of human intestinal organoids using electroporation. Nat Protoc10, 1474-1485 (2015). 
49. Fujii, M. et al. A Colorectal Tumor Organoid Library Demonstrates Progressive Loss of Niche Factor Requirements during Tumorigenesis. Cell Stem Cel/18, 827-838 (2016).

50. Sunami, K. et al. Feasibility and utility of a panel testing for 114 cancer-associated genes in a clinical setting: A hospital-based study. Cancer Sci110, 1480-1490 (2019).

51. Livak, K.J. \& Schmittgen, T.D. Analysis of relative gene expression data using real-time quantitative PCR and the 2(-Delta Delta C(T)) Method. Methods25, 402-408 (2001).

52. Zhou, Y. et al. Metascape provides a biologist-oriented resource for the analysis of systems-level datasets. Nat Commun10, 1523 (2019).

53. Kanda, Y. Investigation of the freely available easy-to-use software 'EZR' for medical statistics. Bone Marrow Transplant48, 452-458 (2013).

\section{Tables}

Due to technical limitations, table 1 is only available as a download in the Supplemental Files section.

\section{Figures}



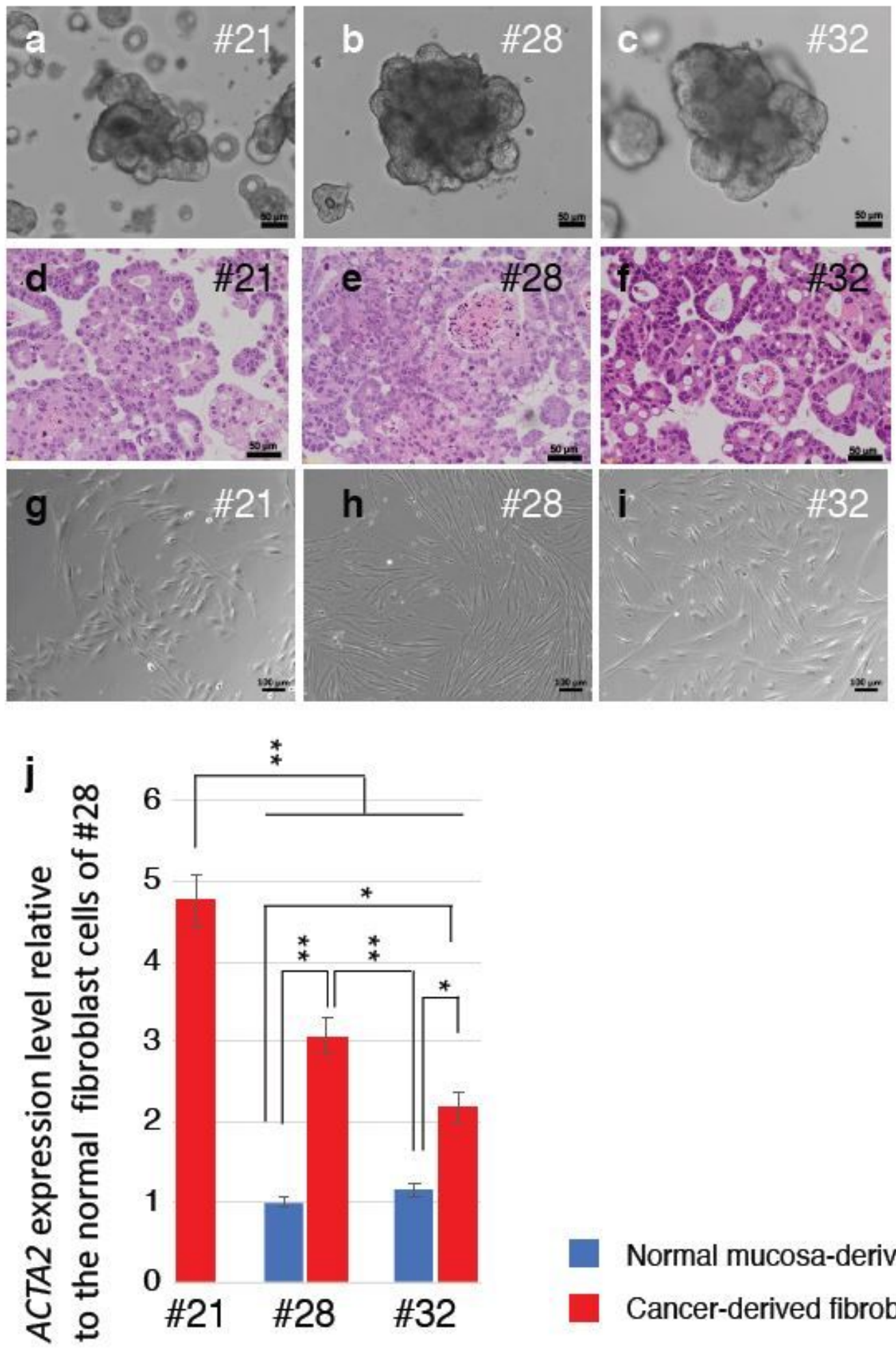

- Normal mucosa-derived fibroblasts

- Cancer-derived fibroblasts

\section{Figure 1}

Morphological and gene expression features of CRC organoids and CAFs (a-c) Bright-field microscopy images of CRC organoids. Scale bar, $50 \mu \mathrm{m}$. (d-f) Representative H\&E micrographs of CRC organoids. Scale bar, $50 \mu \mathrm{m}$. (g-i) Bright-field microscopy images of fibroblasts established from CRC. Scale bar, 100 $\mu \mathrm{m}$. (j) Increased expression levels of ACTA2 in CAFs are shown by comparing with NFs. Blue and red bars represent the relative expression level of ACTA2 in CAFs and NFs, respectively. *: $p<0.05, * *: p<0.01$ 


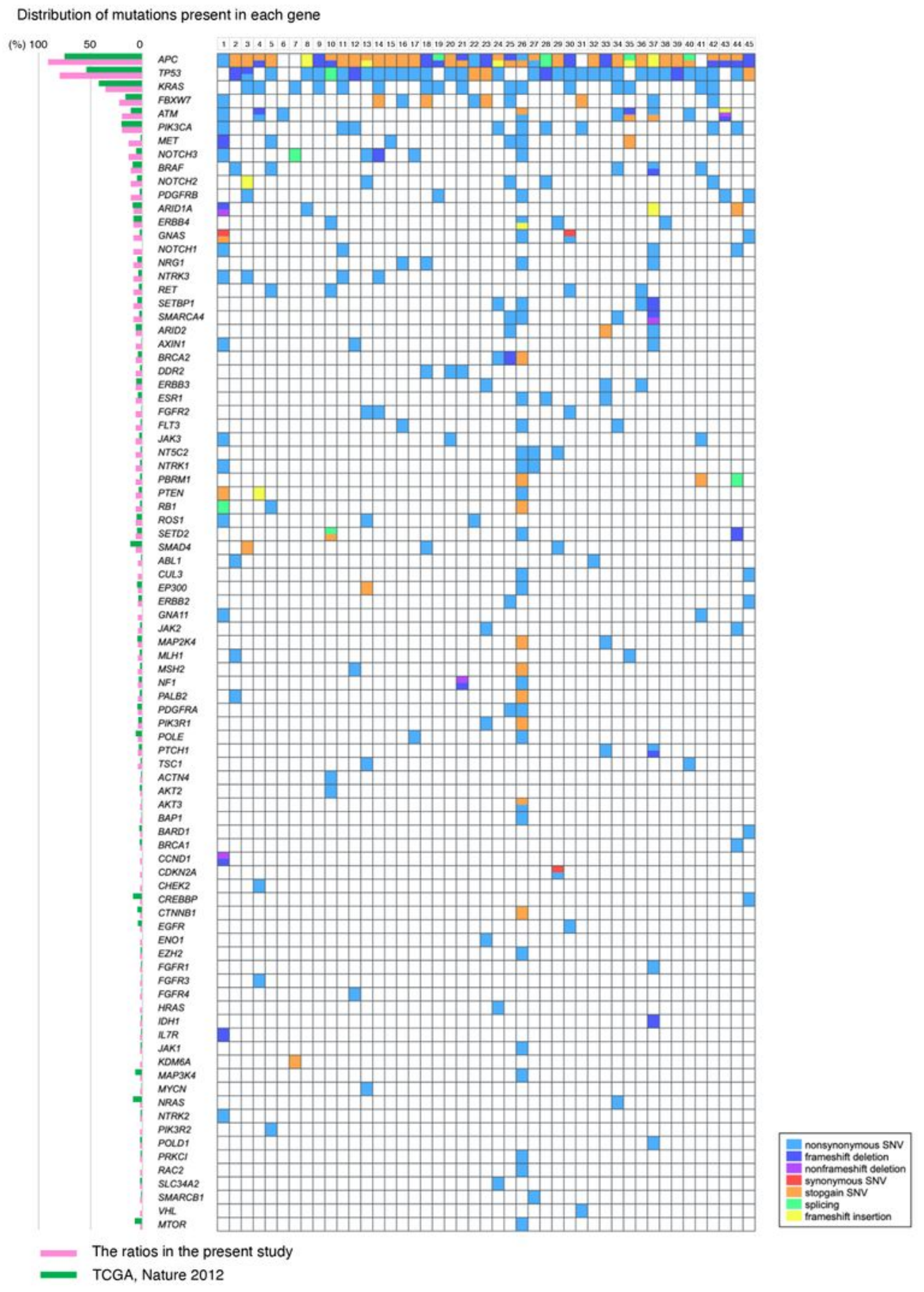

\section{Figure 2}

Comparison chart of gene mutations in 45 CRC samples Mutations of 114 cancer-related genes were analyzed using NCC Oncopanel test. The left graph shows the mutation frequency of each gene in the present 45 samples (pink) and the TCGA data set (green). Most frequently mutated gene was APC followed by TP53 and KRAS. Light blue; nonsynonymous SNV, blue; frameshift deletion, purple; 
nonframeshift deletion, red; synonymous SNV, orange; stopgain SNV, green; splicing, yellow; frameshift insertion.
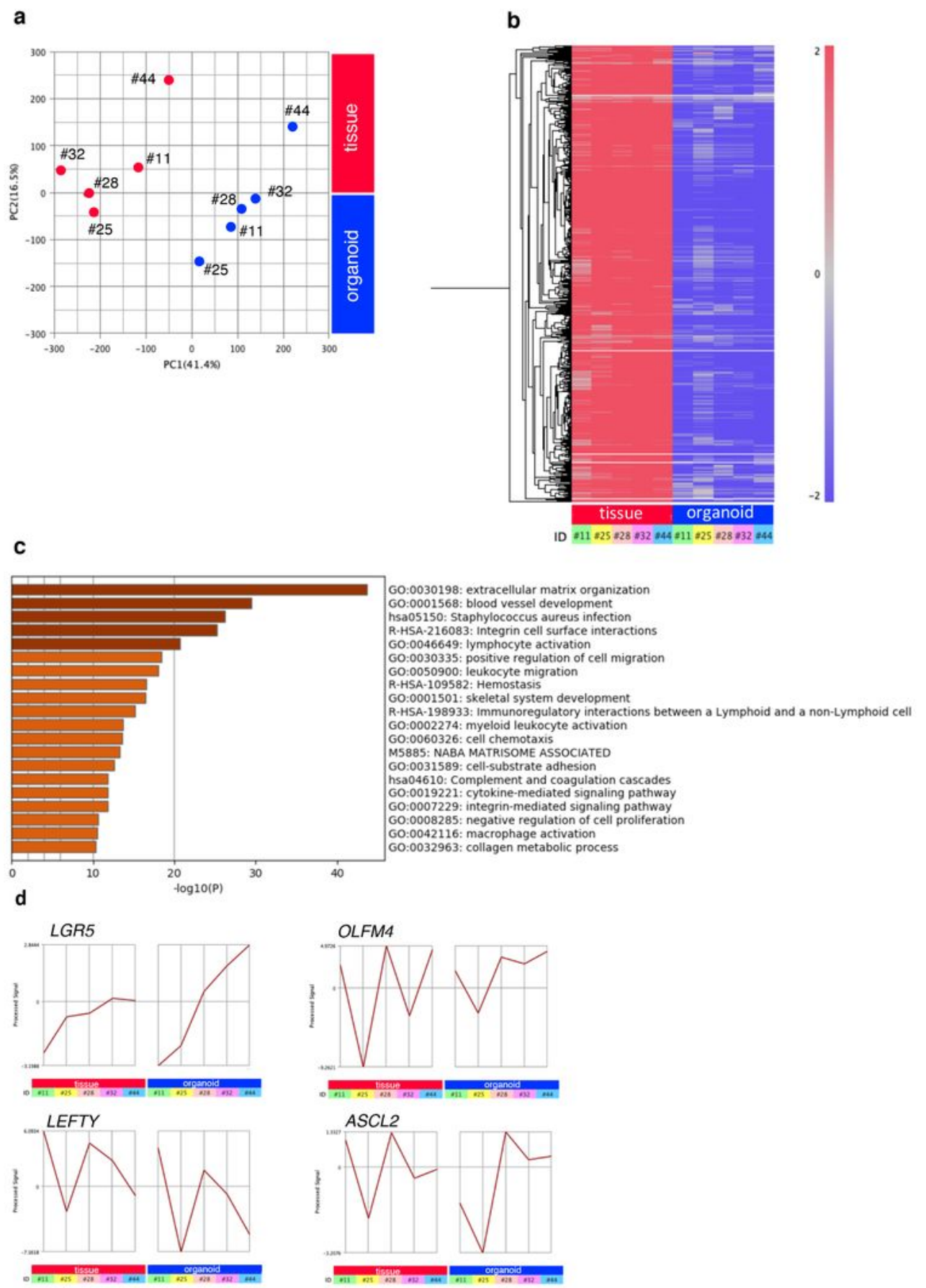

\section{Figure 3}

Comparative gene expression profile between original tumor tissues and organoids. PCA score plot displaying the distinct dissimilarities between the primary tumors and organoids from cases \#11, \#21, \#25, \#28, \#32, and \#44 (a). The PCA scores for components 1 and 2 were determined from the entire 
spectra. (b) In total, 586 probes with a gene expression profile similar to that with high expression only in tumor tissues in PC1 were extracted (Pearson's correlation between 0.95 and 1). Tree clustering of these genes is shown. The clustering separated each sample type. (c) Gene Ontology analysis and significantly enriched GO terms of 586 probes whose expression in organoids was lower than that in the original tumors. (d) Expression profiles of intestinal stem cell marker genes. Line graphs using normalized microarray data show expression levels of LGR5, OLFM4, LEFTY, and ASCL2.

a

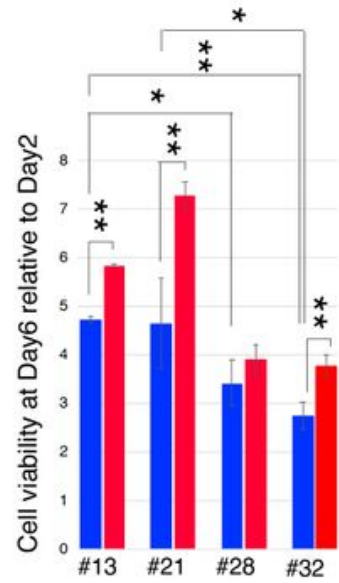

single culture

co-culture

b

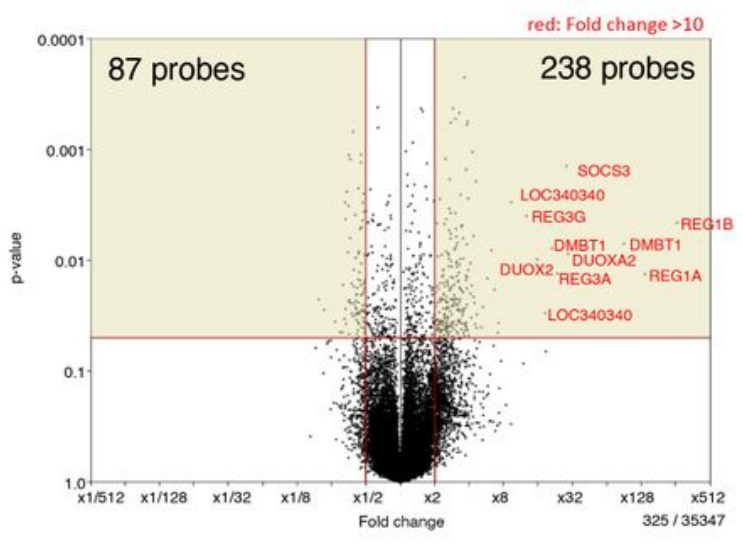

c

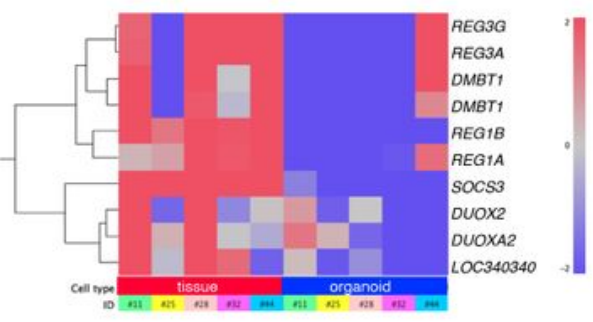

d

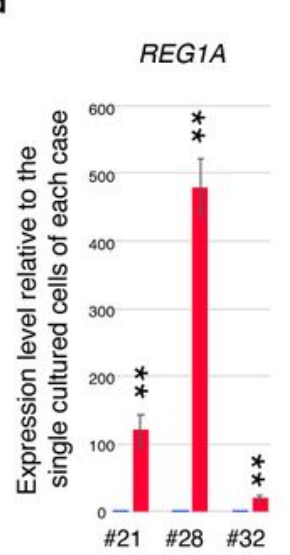

e

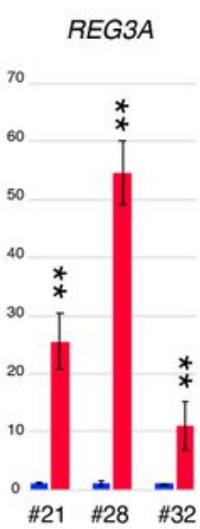

f

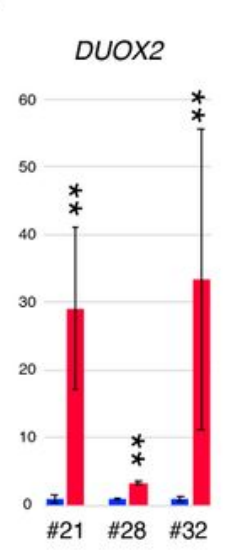

g

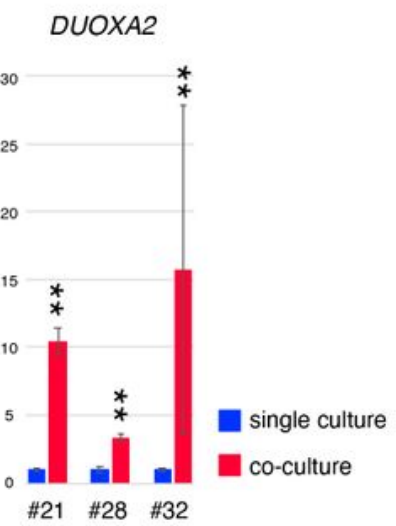


Comparison of cell viability and gene expression profile between organoids with or without co-culture with CAFs Cell viability of single-culture and co-cultured organoids at Day 6. (b) Volcano plot of gene expression levels comparing those in single-culture organoids and those in organoids co-cultured with CAFs $(n=3)$. The $y$-axis shows the P-value for the differences in gene expression levels between organoids with and without co-culture with CAFs by a negative logarithm. The $x$-axis is the log2 difference in the estimated relative gene expression values. Vertical red lines represent the threshold of the log 2-fold change (equivalent to a 2 -fold change). Thus, the dots in the beige-colored region correspond to genes that show a significant $(p<=0.05) 2$-fold or greater change in gene expression between single-culture organoids and organoids co-cultured with CAFs. (c) Gene expression profile of CAF-induced genes with 10-fold upregulation, including REG family genes and DUOX gene families, were extracted from microarray data for CRC tissues and single-culture organoids. Relative quantification of two CAF-induced $\begin{array}{lllll}\text { gene candidates, REG1A } & \text { (d), REG3A } & \text { (e) DUOX2 (f), and DUOXA2 } & \text { (g) measured by qRT-PCR. The }\end{array}$ expression levels of each genes were calculated by comparing with beta-ACTIN. Blue bars represent gene expression levels in organoids without CAFs. Red bars represent gene expression levels in organoids cocultured with CAFs. ${ }^{*}: p<0.01$ 
a

\#28 Organoid
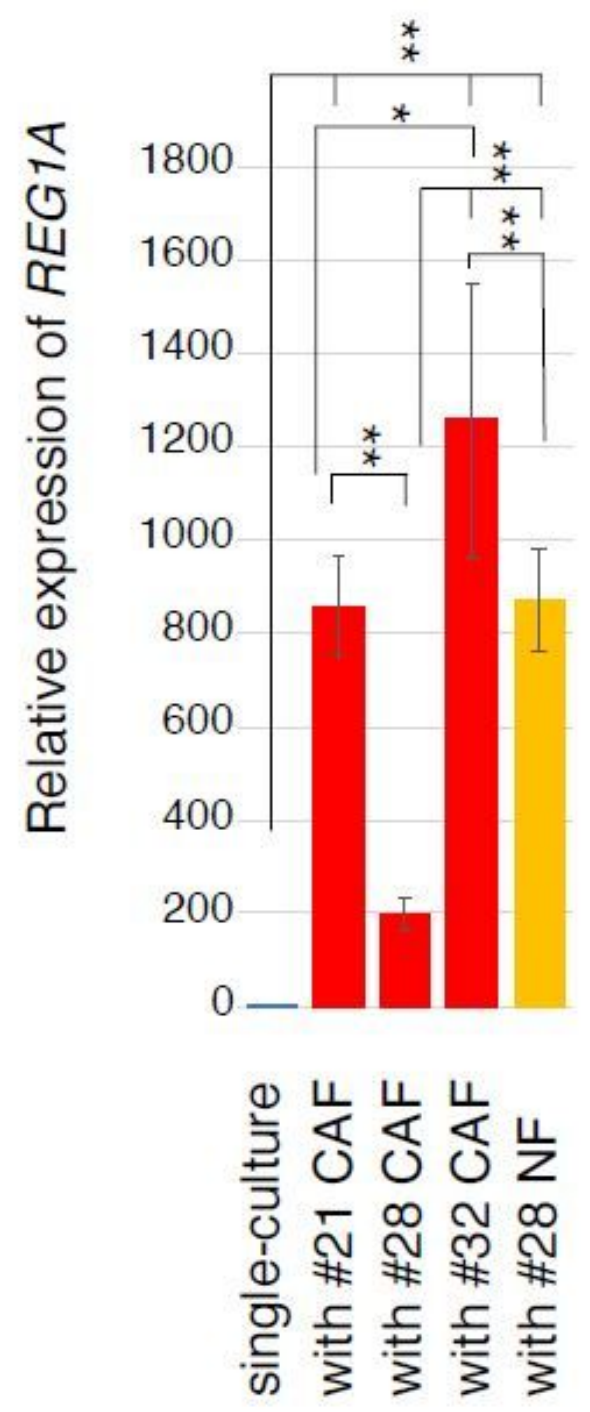

b

\section{\#32 Organoid}

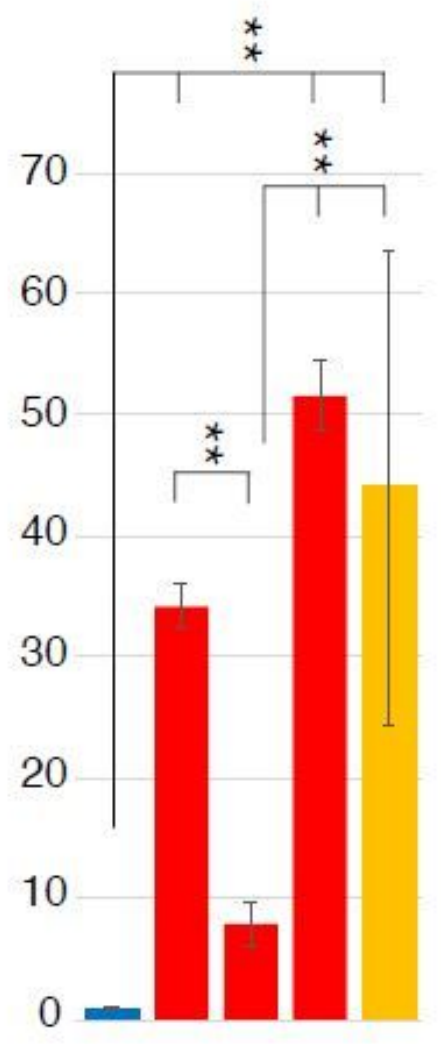

single culture

co-culture with CAF co-culture with NF

\section{Figure 5}

Induction of REG1A in organoids by co-culture with CAFs from three different cases and normal fibroblasts from one case qRT-PCR for REG1A gene in organoids co-cultured with CAFs from three different cases and normal mucosa-derived fibroblasts was performed using \#28 (a) and \#32 organoids (b). Relative gene expression levels are shown with the expression level of single-culture organoids set to 1. *: $p<0.05, * *: p<0.01$

\section{Supplementary Files}


This is a list of supplementary files associated with this preprint. Click to download.

- Table1.pdf

- Supplementv5.pdf 